\title{
Formation of Quantum Dots with Post-growth Thermal Annealing of InGaN/GaN Quantum Wells
}

\author{
Yen-Sheng Lin ${ }^{1}$, Kung-Jen $\mathrm{Ma}^{2}$, Yi-Yin Chung ${ }^{3}$, Chih-Wen Liu ${ }^{3}$,Shih-Wei Feng ${ }^{3}$, Yung-Chen Cheng ${ }^{3}$, \\ C. C. Yang ${ }^{3}$, Cheng-Ta Kuo ${ }^{4}$ and Jian-Shihn Tsang ${ }^{4}$ \\ ${ }^{1}$ Department of Electrical Engineering, Chinese Naval Academy (Taiwan), Kaohsiung, Taiwan \\ ${ }^{2}$ Department of Mechanical Engineering, Chung Hua University, Hsinchu, Taiwan \\ ${ }^{3}$ Graduate Institute of Electro-Optical Engineering and Department of Electrical Engineering, \\ National Taiwan University, 1, Roosevelt Road, Sec. 4, Taipei, Taiwan \\ (phone) 886-2-23657624 (fax) 886-2-23652637 （E-mail) ccy@cc.ee.ntu.edu.tw \\ ${ }^{4}$ Advanced Epitaxy Technology Inc., Hsinchu Industrial Park, Taiwan
}

\begin{abstract}
It is shown that post-growth thermal annealing of such a sample with temperature ranging from 800 to $900{ }^{\circ} \mathrm{C}$ led to a better confinement of indium rich clusters near InGaN quantum well layers. Transmission electron microcopy (TEM) and energy filter TEM results manifested that the sizes of indium-rich QDs were reduced with increasing annealing temperature. Also, the size homogeneity was improved. Quasi-regular arrays of indium-rich QDs embedded in InGaN quantum wells were observed in the sample of $900{ }^{\circ} \mathrm{C}$ annealing. X-ray diffraction also showed the enhancement of InN relative intensity. Photoluminescence measurements revealed blue shifts of photon emission spectral peak, indicating stronger quantum confinement after thermal annealing.
\end{abstract}

Keywords: InGaN/GaN quantum wells, quantum dots, thermal annealing, transmission electron microscopy

\section{INTRODUCTION}

It has been shown that the indium-rich clustering structures have the sizes in the range of a few $\mathrm{nm}$ [1]. The formation of such quantum dot (QD) structures may arise from several mechanisms, including strain induced island formation [2,3], spinodal decomposition [4-6], and surface segregation effect [7]. Efforts have been made to tune photon emission spectra by increasing indium contents in InGaN/GaN QWs. However, it usually resulted in severe compositional fluctuation and larger indium-rich clusters due to the effect of spinodal decomposition [1,8]. Self-assembled QDs grown with MOCVD are more promising. Unfortunately, QDs formed with the Stranski-Krastanow growth mode tend to be randomly distributed across the surface. The optimized conditions for the strain-induced vertical self-alignment of InN QDs are still not mature. Another approach, in which the dot nucleation sites are controlled by patterning the substrate before deposition, seems to be a solution. Nevertheless, it requires multiple steps of lithography and etching, making its translation into mass production difficult. Therefore, it is important to develop a simple and reliable process for manipulating the QD structures in InGaN/GaN QWs. In this paper, we developed a different approach for regular QD formation in InGaN/GaN QWs. It was accomplished by post-growth thermal annealing of samples. After the annealing process, the regularities of size and distribution of indium-rich clusters were significantly improved. X-ray diffraction (XRD), high-resolution transmission electron microscopy (HRTEM) and energy filter transmission electron microscopy (EFTEM) were used to characterize material properties. Also, photoluminescence (PL) measurements demonstrated stronger quantum confinement of the QDs.

\section{EXPERIMENTAL PROCEDURES}

The sample used in this study was grown with a low-pressure metal-organic chemical-vapor deposition (MOCVD) reactor. The InGaN/GaN QW sample consisted of ten periods of InGaN wells with $35 \AA$ in thickness. The designated indium content of the sample was about $19 \%$. The barrier was $100 \AA \mathrm{GaN}$. In the sample, the QW layers were sandwiched between a $1.5 \mu \mathrm{m} \mathrm{GaN}$ buffer layer on a (0001) sapphire substrate and a $50 \mathrm{~nm} \mathrm{GaN}$ cap layer. The growth temperatures were 1020 and $720{ }^{\circ} \mathrm{C}$ for $\mathrm{GaN}$ and $\mathrm{InGaN}$, respectively. As-grown samples were subjected to thermal annealing in a quartz tube furnace at different temperatures ranging from 800 to $950{ }^{\circ} \mathrm{C}$ in nitrogen ambient for $30 \mathrm{~min}$.

Cross-sectional samples were prepared in the conventional manner by grinding, dimpling and $\mathrm{Ar}^{+}$-ion milling with $6 \mathrm{KV}, 1 \mathrm{~mA}$ and an incident angle of $4^{\circ}$. Based on previous experiences with compounds containing 
indium, the ion-milling step was carried out with the specimen holder at the liquid nitrogen temperature in order to minimize ion beam damage. HRTEM investigations were performed with both $200 \mathrm{KeV}$ Philips CM 200 and $300 \mathrm{KeV}$ JEM 3010 microscopes. All the high-resolution micrographs were taken at Scherzer defocus and the samples were viewed along the [11-20] zone axis. The $300 \mathrm{KeV}$ JEM 3010 microscope is equipped with a $2 \mathrm{k} \times 2 \mathrm{k}$ slow-scan CCD camera and a Gatan Imaging Filter (GIF). Energy filtering (EF) images were recorded by using the cross correlation algorithm available in the Digital Micrograph software of the GIF. To remove the background contribution underneath the ionization edges and to obtain elemental maps, the jump-ratio method was used to avoid noise and artifacts. XRD measurements were carried out using $\mathrm{Cu} \mathrm{K}$ radiation, monochromated with the (111) reflection of Ge single crystal.

\section{RESULTS AND DISCUSSIONS}

Figures 1(a)-(d) show the cross-sectional HRTEM bright field images of the as-grown and annealed samples. The variations of the contrast in the pictures represent the fluctuations of indium composition. The diffusive InGaN/GaN QW interfaces can be seen in Fig. 1(a) (the as-grown sample). The average size of the disk-like indium-rich clusters is larger than $10 \mathrm{~nm}$. The clusters are irregularly dispersed and extended into $\mathrm{GaN}$ barrier layers. Post-growth annealing at temperature over $800{ }^{\circ} \mathrm{C}$ led to better confinement of indium-rich clusters near InGaN QWs, as shown in Figs. $1(\mathrm{~b})\left(800{ }^{\circ} \mathrm{C}\right)$ and $(\mathrm{c})\left(900{ }^{\circ} \mathrm{C}\right)$. The QDs become sphere-like shaped and their average size becomes smaller after annealing treatment. Also, their size homogeneity was improved. Interestingly, one can observe that fine indium-rich QDs with size 2-5 nm were regularly distributed within the designated $\mathrm{InGaN}$ QW layers after annealing treatment at $900{ }^{\circ} \mathrm{C}$ (Fig. 1(c)). The originally diffusive cluster boundaries became sharpened after post-growth thermal annealing. However, further increasing annealing temperature to $950{ }^{\circ} \mathrm{C}$ led to over-growth of QDs. In this situation, it is difficult to resolve the InGaN QW structure, as shown in Fig. 1(d).

To further understand the composition variation, EFTEM observation was performed for the samples after annealing treatments. Indium map and indium composition profiles along and across QW layers of the sample after $900{ }^{\circ} \mathrm{C}$ annealing are shown in Fig. 2. Regularly arrayed QDs with nearly the same indium concentration at the cores of the QDs can be observed. The irregular distribution of indium-rich clusters and strong compositional fluctuation in the sample after $950{ }^{\circ} \mathrm{C}$ annealing can be again observed in the EFTEM results, as shown in Fig. 3.

The formation of sphere-like QDs upon thermal annealing may arise from two mechanisms: First, clustering of indium atoms along the [0001] direction and anti-clustering of indium atoms in the (0001) plane are energetically favorable due to dipole interaction energy. Second, a disk-like island with a small height/width ratio is practically non-relaxed. A hypothetical island having the shape of a larger height/width ratio is more stable. High temperature annealing facilitates movement of constituent atoms and interface reconstruction, which lead to sphere-like, energetically more stable indium-rich clusters. The elastic interaction between islands is believed to play a crucial role for forming arrays of islands. The system of interacting islands can be regarded as a system of elastic domains, where the minimum of strain energy corresponds to a periodic domain structure. In the InGaN/GaN QW sample, thermal energy with post-thermal annealing at $900{ }^{\circ} \mathrm{C}$ is sufficient to drive the system to a state with lower energy, i.e. a quasi-ordering regime. The period of the arrayed QDs is believed related to the width and nominal content of indium in the InGaN wells. This issue deserves further investigation.

\section{CONCLUSIONS}

In conclusion, we have observed that post-thermal annealing $\left(800 \sim 900{ }^{\circ} \mathrm{C}\right)$ led to a better confinement of indium-rich clusters near InGaN wells in an InGaN/GaN QW structure. The sizes of indium-rich dots were found reduced by increasing annealing temperature. Also, the size homogeneity was improved. Quasi-regular arrays of indium-rich QDs with relatively sharper interfaces embedded in InGaN QWs were observed in the sample after annealing at $900^{\circ} \mathrm{C}$. However, with annealing temperature at $950{ }^{\circ} \mathrm{C}$, all the regular features of indium-rich clusters disappear. Clusters with sizes significantly larger than the QW width were observed. All the observations with HRTEM, EFTEM, XRD, and PL have shown consistent results.

\section{ACKNOWLEDGEMENTS}

This research was supported by National Science Council, The Republic of China, under the grants of NSC 89-2218-E-002-094, NSC 89-2218-E-002-095, and NSC 89-2215-E-002-051.

\section{REFERENCES}

[1] Y. S. Lin, K. J. Ma, C. Hsu, C. C. Liao, S. W. Feng, C. C. Yang, C. C. Chuo, C. M. Lee, and J. I. Chyi, Appl. Phys. Lett. 77, 2998, 2000. 
[2] K. Tachibana, T. Someya, and Y. Arakawa, Appl. Phys. Lett. 74, 383, 1999.

[3] J. Tersoff, Phys. Rev. Lett. 81, 3183, 1998.

[4] R. Singh, D. Dopplapudi, T. D. Moustakas, and L. T. Romano, Appl. Phys. Lett. 70, 1089, 1997.

[5] I. H. Ho and Stringfellow, Appl. Phys. Lett. 69, 2701, 1996.

[6] M. D. McCluskey, L. T. Romano, B. S. Krusor, D. P. Bour, N. M. Johnson, and S. Brennan, Appl. Phys. Lett. 72, 1730, 1998.

[7] N. Grandjean and J. Massies, B. Damilano, P. Vennegues, L. Siodade, and L. Hirsch, Appl. Phys. Lett. 74, 3616, 1999.

[8] Y. H. Kwon, G. H. Gainer, S. Bidnyk, Y. H. Cho, J. J. Song, M. Hansen and S. P. DenBaars, Appl. Phys. Lett. 75, 2545, 1999.
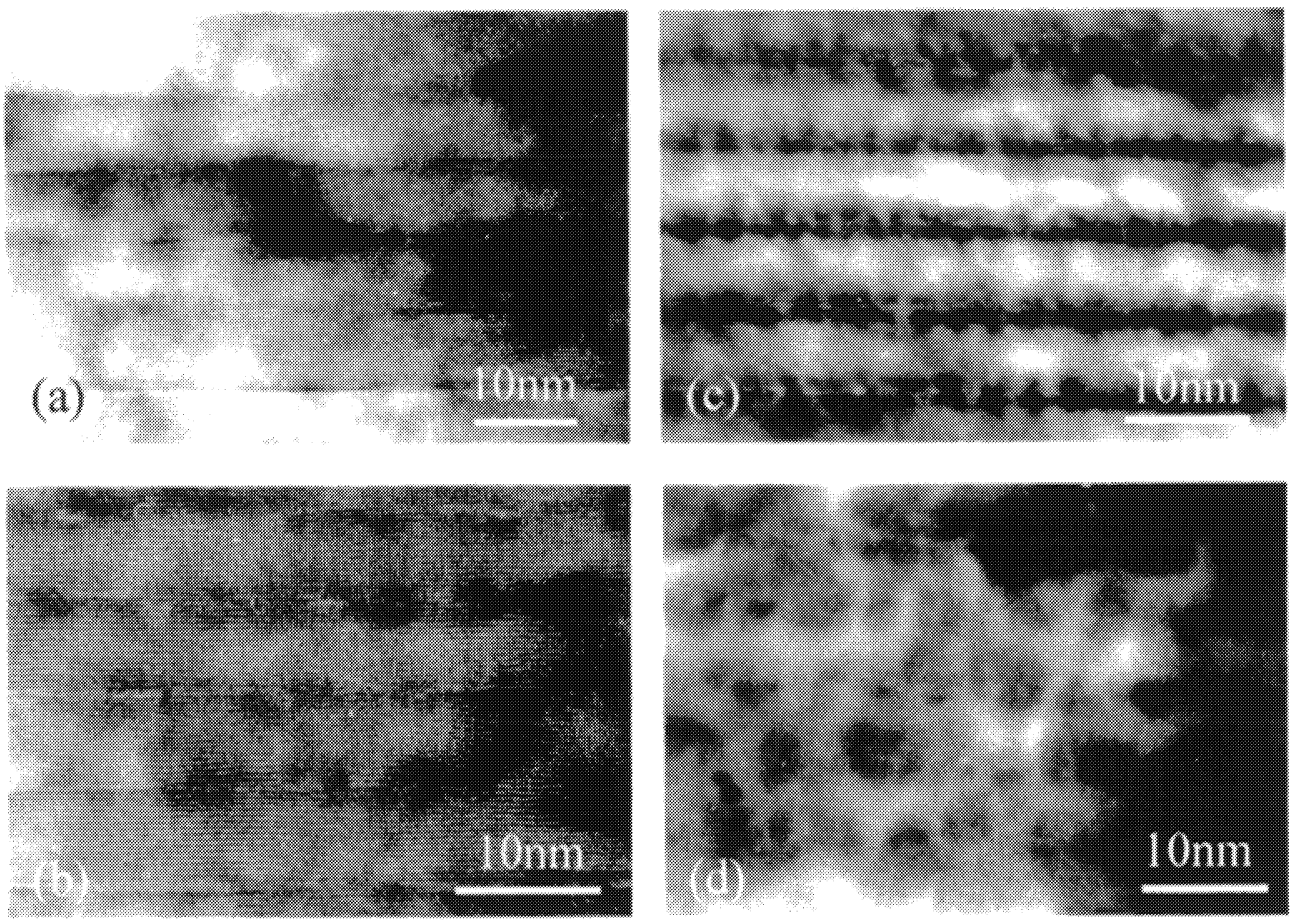

Fig. 1 Cross-sectional HRTEM bright field images of the as-grown and annealed samples: (a) as-grown sample, (b) sample annealed at $850{ }^{\circ} \mathrm{C}$, (c) sample annealed at $900{ }^{\circ} \mathrm{C}$, and (d) sample annealed at $950{ }^{\circ} \mathrm{C}$.

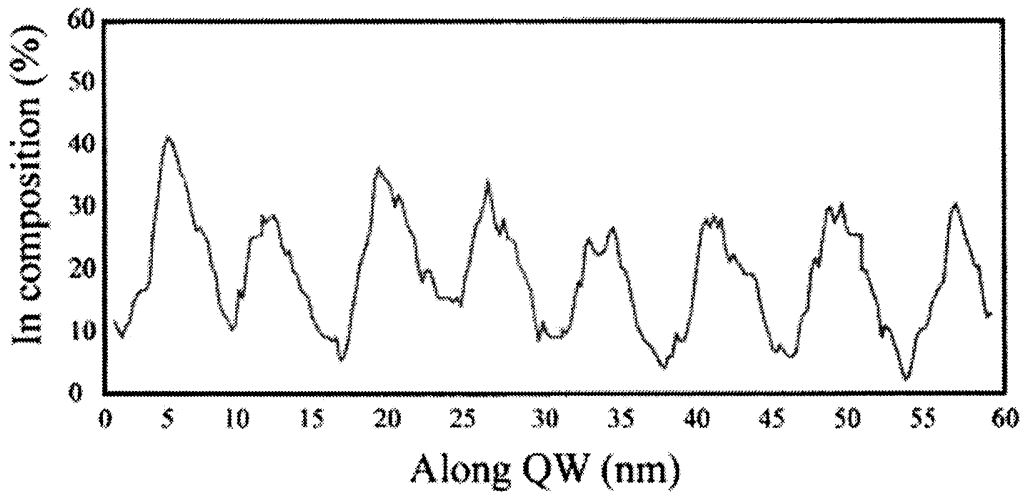

Fig. 2 EFTEM image of the $900{ }^{\circ} \mathrm{C}$ annealing sample showing the indium composition amplitude profile along a QW. 


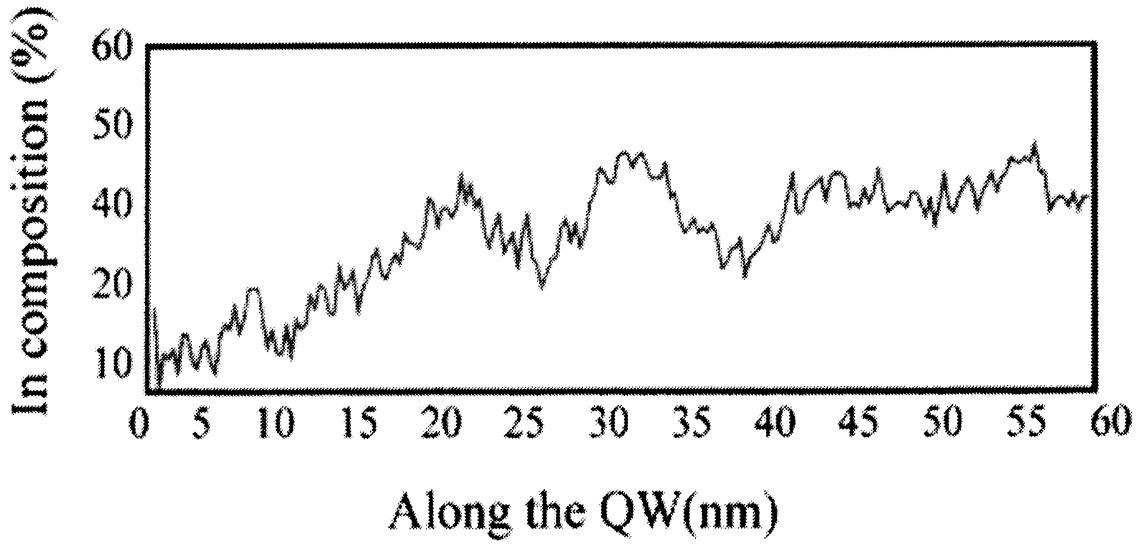

Fig. 3 EFTEM image of the $900{ }^{\circ} \mathrm{C}$ annealing sample showing the indium composition amplitude profile along a QW. 